\title{
Investigations on the detectability of water intruding into bridge deck sealings by electrical resistivity measurements
}

\author{
Carla Driessen ${ }^{1, *}$, Michael Raupach ${ }^{1}$ \\ ${ }^{1}$ Institute of Building Materials Research, RWTH Aachen University, Schinkelstrasse 3, 52062 Aachen, Germany
}

\begin{abstract}
In the context of a current joint research project a monitoring system to detect leakages in bridges is implemented by using a textile reinforced concrete interlayer which consists of two carbon meshes with a spacing of $15 \mathrm{~mm}$ and a new developed mortar. Between the two carbon meshes the resistivity is measured with alternating current. If a leakage in the bridge deck sealing occurs and water gets into the interlayer a drop in the measured resistivity occurs and the leakage is detected. In this case repair measures can be carried out in an early stage or a preventive cathodic protection can be switched on. To assess which sizes of leakages are detectable under which boundary conditions, laboratory tests and tests on a $100 \mathrm{~m}^{2}$ outdoor demonstrator were carried out. Therefore leakages were artificially produced and the changes in the measured resistivity values were observed under different climatic conditions. Impacts such as the form of the leakages or the position of the leakages in the measuring field were varied.
\end{abstract}

\section{Introduction}

\subsection{Electrical resistivity measurement}

Until now, only sensors which selectively measure the electrolyte resistance at the point of installation are available [1, 2]. With Smart-Deck the first full-surface system for the measurement of the mortar resistance is tested [3]. The resistance is calculated according to equation (1).

$\mathrm{R}=\mathrm{U} / \mathrm{I}(1)$

with:

R: electrolyte resistance in $\Omega$

$\mathrm{U}$ : voltage in $\mathrm{V}$

I: current in A

Since the resistance depends on the measuring frequency and the geometry of the specimen, the conversion of an $\mathrm{AC}$ resistance into a resistivity is required which is achieved by using a cell constant according to Eq. (2) [4].

$$
\rho=\mathrm{R}_{\mathrm{el}} \cdot \frac{\mathrm{A}}{1}
$$

A: cross sectional area of the mortar specimen in $\mathrm{m}^{2}$

1: distance between the electrodes in $\mathrm{m}$

\section{Numerical Simulations}

As a first step numerical simulations were carried out to assess which range of leakage sizes are detectable at which mortar resistivities for wet and dry conditions. Therefore mortar resistivities from $100 \Omega$ m up to 1000 $\Omega \mathrm{m}$ were used to represent the leakage zone with soaked mortar. Resistivity values from $1000 \Omega$ m up to 10000 $\Omega m$ represent the dry mortar under the intact sealing. Besides the mortar resistivities and the leakage size, only the depth of the leakage showed a significant influence on the detectability. The simulations showed that the intruding water has almost to reach the second carbon mesh until a decrease in resistivity values is measurable. Other parameters as the quantity of leakages, the form of the leakage and the position of the leakage lead only to negligible changes in the results [5]. As shown in Figure 1 even leakages of a few percentages of the total measuring field lead to a detectable drop in resistivity values. However, it has to be mentioned that boundary conditions such as temperature and humditity were not depicted in the numerical simulations though they will have a high impact on the measured values.

with:

$\rho: \quad$ mortar resistivity in $\Omega \mathrm{m}$

$\mathrm{R}_{\mathrm{el}}$ : measured resistance in $\Omega$

\footnotetext{
* Corresponding author: driessen@ibac.rwth-aachen.de
} 


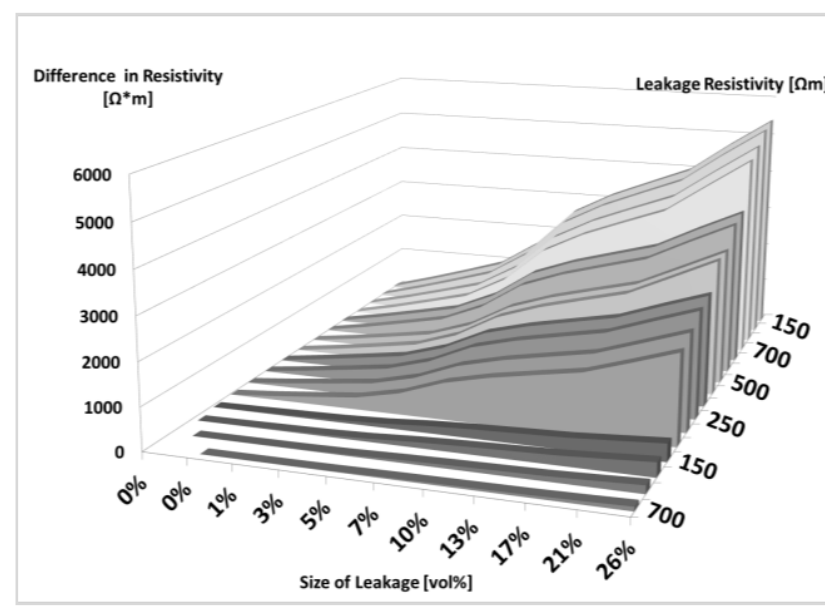

Fig. 1. Difference in resistivity values between initial situation and defective sealing

\section{Laboratory Tests}

\subsection{Pretests}

After having a first benchmark of detectable leakage sizes through the results of the numerical simulations, laboratory tests on specimen with a size of $440 \mathrm{x} 480 \mathrm{x}$ $35 \mathrm{~mm}^{3}$ were performed. The structure followed the original Smart-Deck structure with $35 \mathrm{~mm}$ mortar and two technical carbon meshes with a spacing of $15 \mathrm{~mm}$ in the middle.

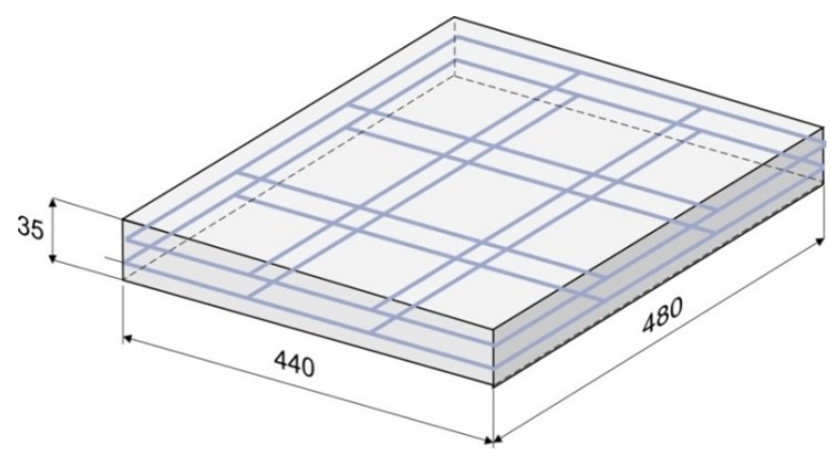

Fig. 2. Draft of a exemplarily mortar specimen with carbon textiles

For a first test measurement the resistivity was measured while putting the specimen completely under water. The following diagram shows the drop in resistance after a few days.

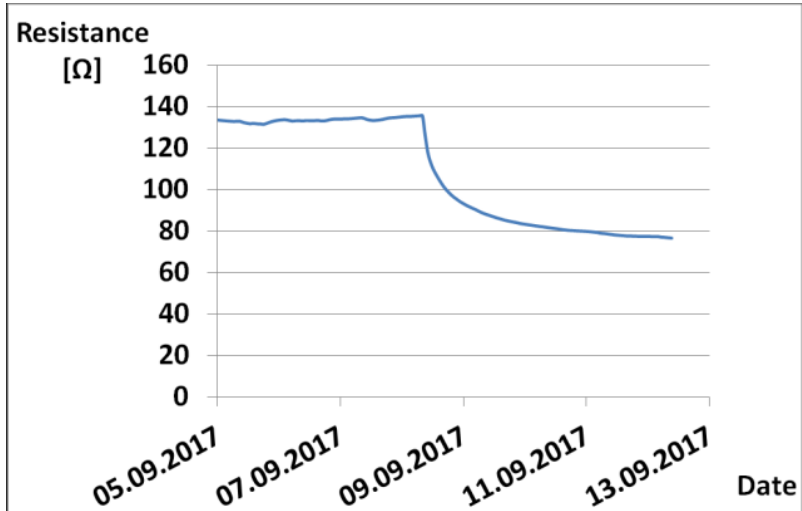

Fig. 3. Behaviour of the resistance measured while putting the specimen under water

Of course this test does not reflect the actual situation of a leakage occurring in a bridge deck sealing and the resulting resistance behaviour. Nevertheless, it could be shown that the concept of a full surface resistance measurement of the concrete using carbon meshes works.

\subsection{Electrochemical impedance spectroscopy (EIS)}

For a more realistic depiction of the detectability of leakages in a bridge deck, smaller leakages and sealed specimens need to be investigated. On top of the specimens artificial leakages were produced by applying sealed water basins on top of the specimen and coating the rest of the concrete surface with epoxy. The water basin, respectively the leakages, varied in their size, form and position on the specimen. Before sealing, the specimens were dried to reach a low water content. After sealing and giving water into the basins, the resistivity measurement started. The resistivity was measured with EIS in a frequency range from $10-10000 \mathrm{~Hz}$.

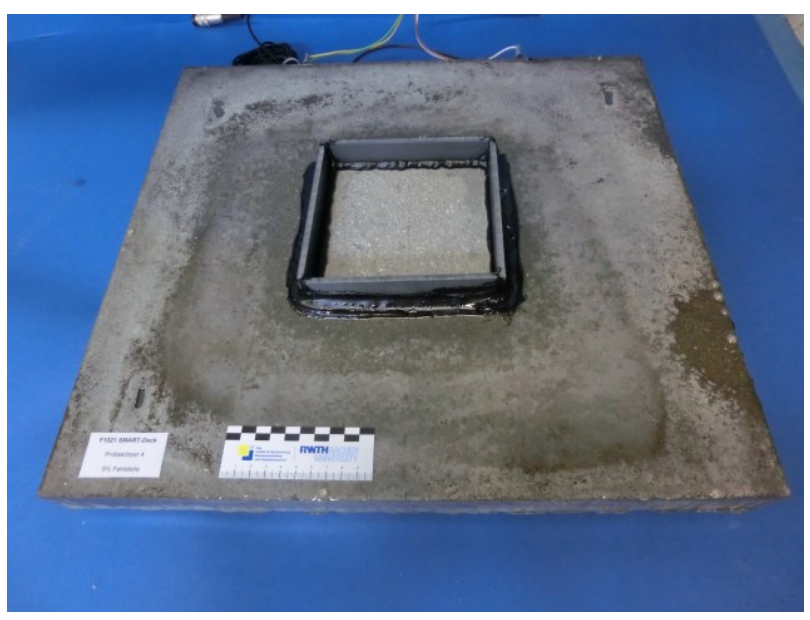

Fig. 4. Exemplary specimen with artificial leakage (water basin) in the middle 
Figure 5 shows an exemplarily plot of the resistivity measurement at $100 \mathrm{~Hz}$. It can be seen that the resistivity values increase over time. The diagram is divided into two parts. In the first time no water was added on top of the specimen to have reference values and then water was filled into the water basin on top of the specimen. Both periods show an increase in resistivity values whereby after some days of water admission the gradient flattens al little bit. This is into contradiction to the expected effect, that the intruding water will cause a decrease in resistivity values. Furthermore it is to mention that the increase in resistivity values is higher at higher frequencies.

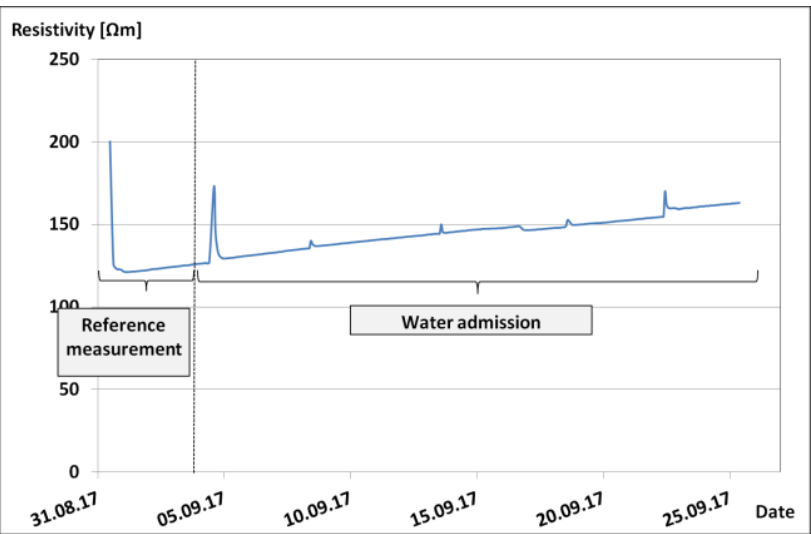

Fig. 5. Plot of the resistivity measurement

\subsection{Nuclear Magnetic Resonance}

There are several possible reasons why the expected decrease in resistance stays out. First it had to be investigated whether water intruded into the Smart-Deck layer at all. Therefore Nuclear Magnetic Resonance was used. Figure 6 shows the moisture distribution in the Smart-Deck layer after 7, 14 and 21 days of water admission. The area between 0.5 and $8.38 \%$ water content is marked with two lines where $0.5 \%$ water content was ascertained by drying the probe in the oven at $110{ }^{\circ} \mathrm{C}$. The water content of $8.38 \%$ was achieved through water saturation. Figure 6 clearly shows higher water contents in the specimen in the upper two layers. So it can be said that water is soaked into the specimen and theoretically a decrease in resistivity values should be measurable.

\subsection{Further laboratory tests}

So further tests were carried out which showed an increase in resistivity values over the time while changing nothing on the specimens (no water admission, too). Under these circumstances it is possible that water admission only leads to a more moderate increase of resistivity rather than to the expected decrease. There are several possible explanations for the increase in resistivity values over time for example:

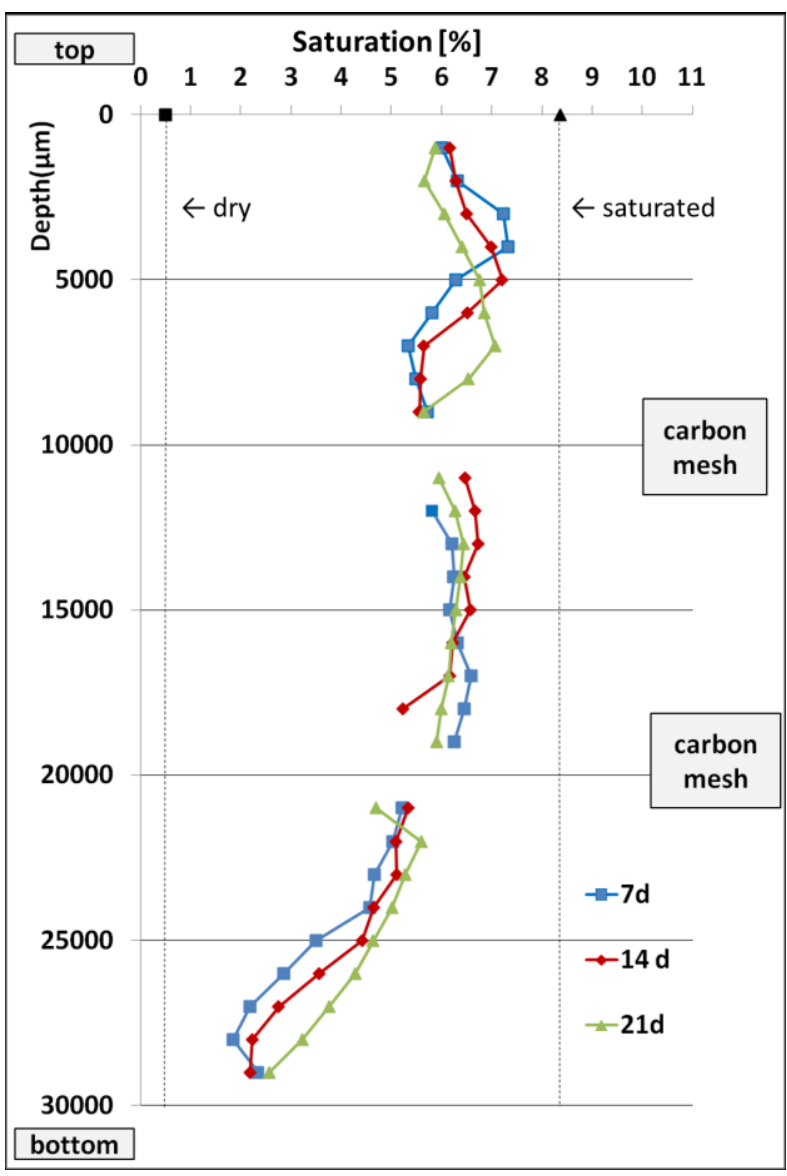

Fig. 6. NMR Results of the water distribution in the Smart-Deck layer

- Effects because of the ongoing hydration of the mortar. With the hydration progress resistivity values increase.

- Effects because of potential gradients between the two carbon meshes (caused by different moisture contents when the intruding water has already reached the first but not yet the second carbon mesh)

- Capacitor effects because of the large electrode surface and the small distance between the electrodes

- Effects caused by contact resistances because of the desiccation behaviour of the textile polymer impregnation. It is possible that the polymer impregnation has a delayed water ad- and desorption behaviour compared to the mortar

The first two options could already be excluded. The first in replicate the tests with more matured specimens and the second in applying an artificial potential gradient with an rectifier and monitoring the resistance values, which were not affected by the potential gradient. So in the next steps the remaining two issues will be investigated.

\section{Tests on a large scale demonstrator}

Beside the laboratory tests, measurements on an outdoor demonstrator with a size of approximately $100 \mathrm{~m}^{2}$ were 
carried out. The demonstrator has four electrically disconnected measuring fields. On each measuring field a different leakage size and form was simulated. Figure 7 shows the demonstrator with the water basins and coated with yellow epoxy.

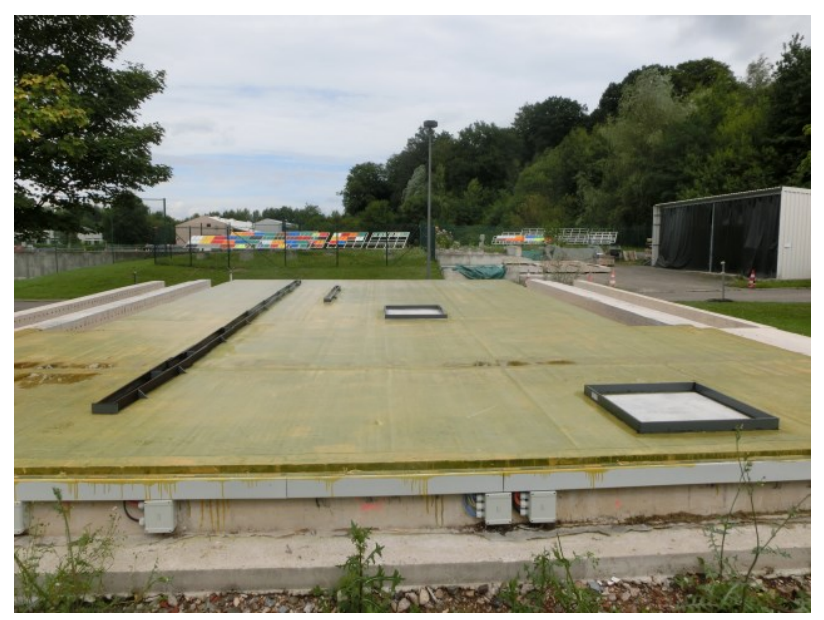

Fig. 7. Outdoor demonstrator

First, over a few months the resistivity values were measured to clarify whether rainfall events can be seen in the resistivity values. Therefore the weather data were compared with the resistivity values. However a significant reaction caused by rainfall could not be seen in the resistivity data. Nevertheless this does not mean that leakages in bridge deck sealings will not be detectable too, because in bridges intruding water will probably accumulate between the bridge sealing and the Smart-Deck layer and the sealing will prevent desiccation. Furthermore it is assumed that the mortar on a bridge will be dried up until leakages in the sealer will occur. So the difference in resistivity values will become clearer. To come closer to the real situation, the water basins were filled completely with water for the second phase. This phase took a few months, too. The following diagram shows the resistivity values, the temperature and the temperature compensated resistivity values.

It can clearly be seen that the resistivity values follow the fluctuations in temperature which means that the measuring technique seems to work reliably. Furthermore it can be said that no short circuit exists between the two carbon meshes. However a distinct decrease in resistivity values triggered by the applied water could not be seen. Again, this could result from the effect that the mortar still had a high water content and was not dried up enough. So the values will be recorded for a longer time period. Otherwise it is possible that without water ingress one would measure an increase in resistivity values as seen in the laboratory tests and that by now this increase is inhibited by the decrease in resistance caused by the water ingress [6].

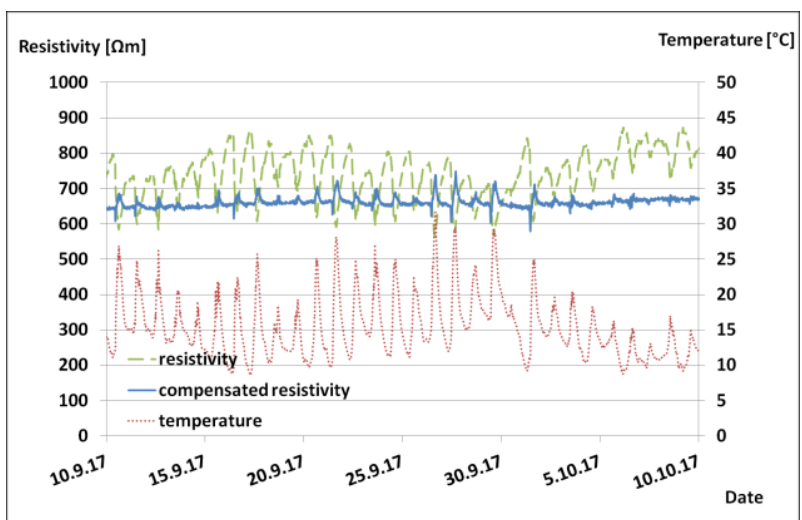

Fig. 8. Course of temperature, resistivity and temperature compensated resistivity of one exemplary measuring field

\section{Conclusion and Outlook}

The following conclusions can be drawn from the described tests:

- According to numerical simulations leakage sizes of a few percentage of the total field size are detectable

- Laboratory tests and tests on an outdoor demonstrator showed that the functionality of the full-surface monitoring in principle works

- The developed mortar shows a good water absorption which is essential for the detectability of leakages

In next steps some tests will be carried out to investigate the effect of increasing resistivity values over time. Therefore specimen will be prepared using titanium meshes as electrode material to investigate whether water absorption and desorption of the coated carbon textiles lead to the increase in resistivity values. Furthermore water saturated carbon textiles with epoxy resin and carbon textiles without resin will be used in specimens to see whether this could prevent the increase of resistivity values.

In 2018 a second demonstrator will be constructed. This time the demonstrator will be applied on a real bridge deck under real traffic conditions.

The authors would like to thank the BMBF for sponsoringthe project and the project executing organization, "VDI Technologiezentrum GmbH (VDITZ)" for the support.

(http://www.eurovia.de/standort/17866-

niederlassung-bauwerksinstandsetzung).

The project partners:

Eurovia Beton GmbH, Niederlassung Bauwerksinstandsetzung,Hofheim-Wallau

(Projektkoordinator);Bundesanstalt für Straßenwesen, Bergisch-Gladbach; FTA GmbH, Albstadt; Massenberg GmbH, NL Bürstadt; Sto SE \& Co. KGaA, Stühlingen;Institut für Massivbau (IMB), RWTH Aachen 


\section{References}

1. Raupach, M. ; Gulikers, J. ; Reichling, K.: «Condition Survey with Embedded Sensors Regarding Reinforcement Corrosion: Bauwerksüberwachung mit eingebetteten Sensoren hinsichtlich der Korrosion von Stahl in Beton ». In: Materials and Corrosion 64 (2013), Nr. 2, S. 141 146 ISSN 1521-4176

2. Raupach, M. ; Dauberschmidt, C. ; Wolff, L.: «Monitoring the Moisture Distribution in Concrete Structures ». London : Taylor \& Francis Group, 2006.- In: Proceedings of the International Conference on Concrete Repair, Rehabilitation and Retrofitting (ICCRRR), Cape Town, South Africa, 21-23 November, 2005, (Alexander, M.G. ; Beushausen, H.-D. ; Dehn, F. ; Moyo, P. (Eds.)), S. 166-167

3. Driessen, C. ; Raupach, M.: Intelligent, Multifunctional Textile Reinforced Concrete Interlayer for Bridges. London [u.a] : CRP Press Taylor \& Francis Group, 2016. - In: Proceedings of the 4th International Conference on Concrete Repair, Rehabilitation and Retrofitting (ICCRRR), Leipzig, 5-7 October 2015, (Dehn, F. ; Beushausen, H.-D. ; Alexander, M.G. ; Moyo, P. (Eds.)) ISBN 978-1-138-02843-2

4. Moosbrugger, C.: Electrical and Magnetic Properties of Metals. ASM International. Materials Properties Database Committee. ISBN: 0-87170690-3

5. Driessen, C. ; Raupach M. «Numerical simulations for the detection of leakages in bridge deck sealers through resistivity measurements $\gg$ in VIII International $\mathrm{PhD}$ Student Workshop on Service Life and Durability of Reinforced Concrete (Springer ; not yet published)

6. Krell, N. «Investigations on the detectability of water intruding into mortar by electrical resistivity measurements » (Master thesis, ibac, RWTH University) 